\title{
Subject Disease Milestones Domain
}

National Cancer Institute

\section{Source}

National Cancer Institute. Subject Disease Milestones Domain. NCI Thesaurus. Code C147184.

A subject domain utilized for the submission of information encompassing and representing data, vocabulary or records related to subject disease milestones. 